\title{
Sinusoidal Movement of a Grating Across the Monkey's Fingerpad: Temporal Patterns of Afferent Fiber Responses
}

\author{
J. W. Morley and A. W. Goodwin \\ Department of Anatomy, University of Melbourne, Parkville, Victoria 3052, Australia
}

Responses were recorded from cutaneous afferents innervating mechanoreceptors in the monkey's fingerpad, while gratings of alternating grooves and ridges were moved sinusoidally across their receptive fields. The gratings were specified by their spatial period and the movement by its peak speed: together these determined the peak temporal frequency at which grating ridges passed over the receptive field. During the central $42^{\circ}$ of each half cycle of movement, the speed and thus the temporal frequency of the grating ridges remained constant to within $6.6 \%$ of their peak values. In this region the responses of all afferents were phaselocked to the temporal sequence of grating ridges. The number of impulses elicited by each grating ridge was a function of the stimulus variables. For all 3 afferent classes-namely, slowly adapting afferents (SAs), rapidly adapting afferents (RAs), and Pacinian afferents (PCs) - the number of impulses per grating ridge increased as the spatial period of the grating increased (while the peak speed of movement was held constant). Similarly, for all 3 classes, the number of impulses per ridge decreased as the peak speed of movement increased (while the spatial period of the grating remained constant). When the peak temporal frequency of the grating ridges was held constant, for SAs and RAs the number of impulses per ridge increased with an increase in the spatial period of the grating and thus with an increase in the peak speed. These phase-locked responses provided information about the peak temporal frequency of the grating ridges independent of the grating spatial period and of the peak speed of movement. The shape of the response profile during a half cycle of movement was different for different afferents. Many of the RA response profiles were close to sinusoidal. The SA and PC profiles tended to have reduced peaks or raised troughs, resulting in flatter profiles. Other departures from sinusoidal profiles were also seen.

When a textured surface is moved sinusoidally back and forth over the fingerpad, the responses of the cutaneous afferents engaged vary with the characteristics of the surface and with the characteristics of the movement. In the previous paper (Goodwin and Morley, 1987a), we used a grating of alternating grooves

\footnotetext{
Received July 9, 1986; revised Dec. 9, 1986; accepted Jan. 8, 1987.

This work was supported by a grant from the National Health and Medical Research Council of Australia. We thank Neil Ratcliffe, Colin Clarke, and Alf Medoro for technical assistance, Kati Bromley for the illustrations, and Janet Bennett for typing the manuscript.

Correspondence should be addressed to Dr. A. W. Goodwin, Department of Anatomy, University of Melbourne, Grattan Street, Parkville, Victoria 3052, Australia.

Copyright (C) 1987 Society for Neuroscience $0270-6474 / 87 / 072181-11 \$ 02.00 / 0$
}

and ridges as a textured surface, and described the afferent responses as a function of the spatial period of the grating and of the peak speed of sinusoidal movement. These 2 parameters together determine the peak temporal frequency at which grating ridges pass over the receptive field, and the afferent responses were also described in terms of this parameter. The measure of response used was the average response over a cycle of movement. This is a very simple measure for the brain to compute, and there are many examples in the literature of neural coding schemes based on simple average responses (Gardner and Spencer, 1972; Johnson et al., 1979; Orban et al., 1979; Phillips and Johnson, 1981). However, the pattern of impulses within a cycle contains much information that could be used by the brain to assist in discrimination or identification of different textured surfaces and different movement profiles.

In a previous study of the responses of cutaneous afferents to gratings moved linearly (constant speed) over the fingerpad (Darian-Smith and Oke, 1980), many of the afferent responses were found to be phase-locked to the temporal sequence of ridges passing over the receptive field. For linear movement, ridges pass over the receptive field at a constant temporal frequency. For certain combinations of stimulus parameters, Darian-Smith and Oke (1980) found that the afferent responses were phaselocked with 1 impulse per grating ridge, while for other combinations the responses were phase-locked with more than 1 impulse per grating ridge or with impulses occurring with only a fraction of the ridges. They proposed that this phase locking was a major factor in the representation of textured surfaces in the brain. Phase locking has been recognized for many years as an important coding mechanism both in the tactile system (Talbot et al., 1968; LaMotte and Mountcastle, 1975; Ferrington and Rowe, 1980) and in the auditory system (Rose et al., 1967). Other aspects of the temporal pattern of neural impulses have been investigated in a wide range of neural systems (Perkel and Bullock, 1968).

When a grating moves sinusoidally back and forth across the fingerpad, both the speed of the movement and the temporal frequency at which ridges pass over the receplive field vary with time. In this paper we investigate the temporal profile of afferent responses, during a cycle of movement, and what information this could provide about the characteristics of the textured surface or about the characteristics of the movement.

\section{Materials and Methods}

Data were collected from 22 slowly adapting afferents (SAs), 30 rapidly adapting afferents (RAs), and 7 Pacinian afferents (PCs). These are the same afferents as reported on in the previous paper (Goodwin and Morley, 1987a), which describes the experimental techniques in detail Briefly, action potentials were recorded from cutaneous afferents while 
a grating of alternating grooves and ridges was moved sinusoidally back and forth over the receptive field. In the case of SAs and RAs the receptive fields were located centrally on a fingerpad, while for PCs the receptive fields could be assigned to a single finger and sometimes to the proximal phalanges, responding vigorously to gratings moved over the fingerpad. The grating was tangential to the fingerpad at the center of the receptive field and was indented $1 \mathrm{~mm}$ into the finger from the point of contact.

\section{Results}

The ratio of the groove width to ridge width of the gratings was held constant at a nominal value of 7 so that the textured surface was completely specified by the grating spatial period, which had nominal values of $0.75,1,1.25,1.5,1.75,2$, or $3 \mathrm{~mm}$. The movement of the grating was sinusoidal with a peak-to-peak amplitude of $80 \mathrm{~mm}$ so that the movement was completely specified by a single parameter, chosen for convenience as the peak speed.

As the grating moved over the receptive field, successive ridges moved over the same point in the receptive field in a specific temporal sequence. This sequence was determined by both the spatial period of the grating and the peak speed of movement as derived in the Appendix.

\section{Phase locking at maximum grating speed}

At the 2 points in the cycle where the grating reached maximum speed (the midpoint of movement in the forward direction and the midpoint of movement in the backward direction), the speed remained constant to within $6.6 \%$ of its peak value $s$ over a $42^{\circ}$ portion of the cycle (see Fig. 5A). Thus, as shown in the Appendix, here the temporal frequency of the ridges was constant to within $6.6 \%$ of its peak value $f$ and was determined by the spatial period $p$ of the grating and the peak speed of movement $s$ of the grating according to the relationship $f=s / p$. The $42^{\circ}$ segments were easy to identify as the stimulator produced a TTL pulse at every $6^{\circ}$ of the cycle.

Figure $1 A$ illustrates the response of an RA to a grating with a spatial period of $2 \mathrm{~mm}$ moved sinusoidally over the receptive field with a peak speed of $80 \mathrm{~mm} / \mathrm{sec}$. The broken lines demarcate the first $42^{\circ}$ portion of the cycle, during which the grating speed was constant to within $6.6 \%$ of $80 \mathrm{~mm} / \mathrm{sec}$. Here, the temporal frequency of the grating ridges was constant to within $6.6 \%$ of the peak value of $40 \mathrm{~Hz}$, and the interval between successive ridges $(25 \mathrm{msec}$ ) is represented by the arrowheads on the time axis below the ensembles of impulses. The first arrowhead has been arbitrarily lined up with the occurrence of the first action potential in the first ensemble. It is clear from the ensembles that during this portion of the cycle the response of the afferent was phase-locked to the grating ridges with 1 impulse occurring for each grating ridge. The precision of the phase locking can be seen in the interspike interval histogram at the top right of the figure. This histogram shows the distribution of intervals between successive action potentials occurring during the $42^{\circ}$ portion of the cycle. The distribution of 154 intervals has a mean value of $25.9 \mathrm{msec}$ and a SD of $1.3 \mathrm{msec}$. A simple method of demonstrating the presence of periodic signals is to compute the autocorrelation function, which, in the case of trains of neural impulses, can be approximated by the expectation density histogram (Poggio and Viernstein, 1964). The autocorrelation function at the lower right of Figure $1 A$ also shows that the $25 \mathrm{msec}$ interval between grating ridges is clearly reflected in the temporal sequence of action potentials. Precise phase locking with 1 impulse per grating ridge, as illustrated in Figure $1 A$, was seen in all 3 types of afferent under certain stimulus conditions. (We use impulses per ridge as a convenient shorthand for impulses per spatial cycle of the grating and do not impute responses to the ridge rather than to the groove.)

Figure $1 B$ illustrates the response of an SA to a $3 \mathrm{~mm}$ grating moved over the receptive field at a peak speed of $120 \mathrm{~mm} / \mathrm{sec}$. Thus, the peak temporal frequency of the ridges was $40 \mathrm{~Hz}$, and the corresponding interval between ridges was $25 \mathrm{msec}$. The figure is similar to Figure $1 A$, and in this case the ensembles of impulses show that the afferent's response was phase-locked to the grating ridges, with 3 impulses occurring with each ridge. Thus, the interspike interval histogram at the top right of Figure $1 B$ does not contain an unambiguous reflection of the interval between grating ridges. However, the autocorrelation function or expectation density histogram at the lower right of Figure $1 B$ does unambiguously show the interval between grating ridges. The autocorrelation function is a mathematical statement of a fact that is clearly seen in the ensembles of Figure $1 B$ : despite the occurrence of 3 impulscs for cach grating ridge, the brain can easily extract the interval between successive grating ridges or, alternatively, the temporal frequency of the ridges. This type of phase locking with multiple impulses per grating ridge was commonly seen in all 3 afferent types. The triple impulses also result in a peak of 120 impulses/sec in the poststimulus time histogram (PSTH) of Figure $1 B$, as compared with the peak value of 40 impulses/sec in Figure $1 A$.

The responses of an RA to a grating with a $1 \mathrm{~mm}$ spatial period moved over its receptive field with a peak speed of 40 $\mathrm{mm} / \mathrm{sec}$ are illustrated in Figure $1 \mathrm{C}$. During the central $42^{\circ}$ of the cycle, the temporal frequency of the ridges was $40 \mathrm{~Hz}$, with a corresponding interval of $25 \mathrm{msec}$. From the ensembles of impulses it is clear that the afferent's response was phase-locked to the ridges with an impulse occurring with only some of the ridges. Again the interspike interval histogram on the top right

\footnotetext{
Figure 1. Phase-locked responses. $A$, An RA responding to a $2 \mathrm{~mm}$ grating moving over the receptive field with a peak speed of $80 \mathrm{~mm} / \mathrm{sec}$ (peak temporal frequency of the ridges, $40 \mathrm{~Hz}$ ). Top left, Speed profile during 1 cycle; below, the cycle histogram averaged over 11 cycles. Broken lines in the histogram show the $42^{\circ}$ segment, during the first half cycle, when speed was constant to within $6.6 \% \mathrm{of} 80 \mathrm{~mm} / \mathrm{sec}$. In the ensembles at bottom left, for 4 consecutive cycles, each stroke shows the time of occurrence of an action potential with the time scale expanded to cover only the $42^{\circ}$ segment. Arrows show the time separation between successive grating ridges $(25 \mathrm{msec})$, with the first arrow arbitrarily aligned with the first impulse in the first ensemble. Responses were phase-locked with 1 impulse per grating ridge. Top right, Interspike interval histogram (bin width $2 \mathrm{msec}$; ordinate is mean number of intervals over the 11 cycles). Bottom right, Autocorrelation or expectation density histogram (bin width 2 $\mathrm{msec}$; ordinate gives the probability of an impulse occurring). $B$. An SA responding to a $3 \mathrm{~mm}$ grating moving over the receptive field with a peak speed of $120 \mathrm{~mm} / \mathrm{sec}$ (peak temporal frequency of the ridges, $40 \mathrm{~Hz}$ ). Format as in $A$. Responses were phase-locked with 3 impulses per grating ridge. Number of cycles is 11 , bin widths are 2 and $4 \mathrm{msec}$ for interspike interval and autocorrelation histograms, respectively. $C$, An $R A$ responding to a $1 \mathrm{~mm}$ grating moving over the receptive field with a peak speed of $40 \mathrm{~mm} / \mathrm{sec}$ (peak temporal frequency of the ridges, $40 \mathrm{~Hz}$ ). Format as in $A$. Responses were phase-locked with impulses occurring with only some of the grating ridges. Number of cycles is 10 , bin widths are 2 and 4 msec for interspike interval and autocorrelation histograms, respectively.
} 
A.

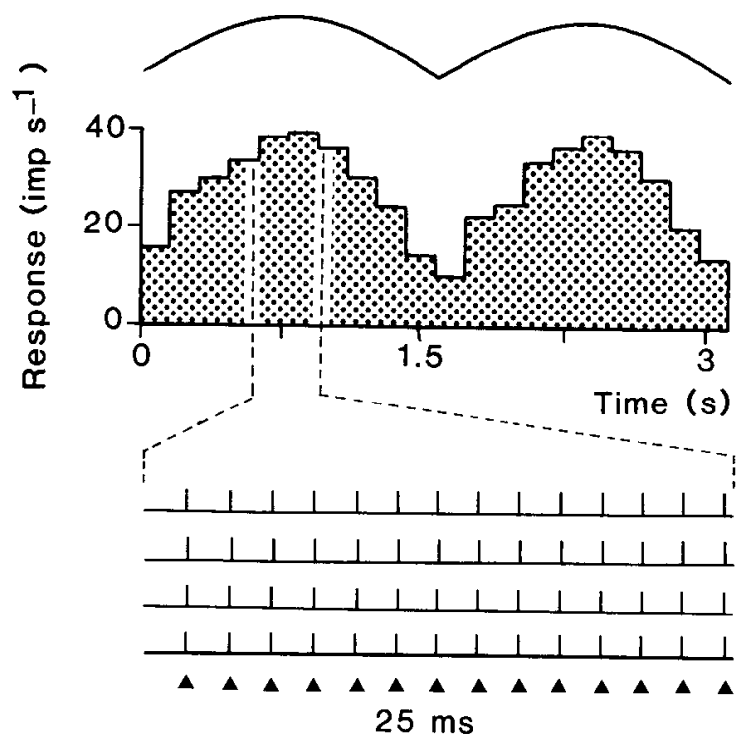

B.

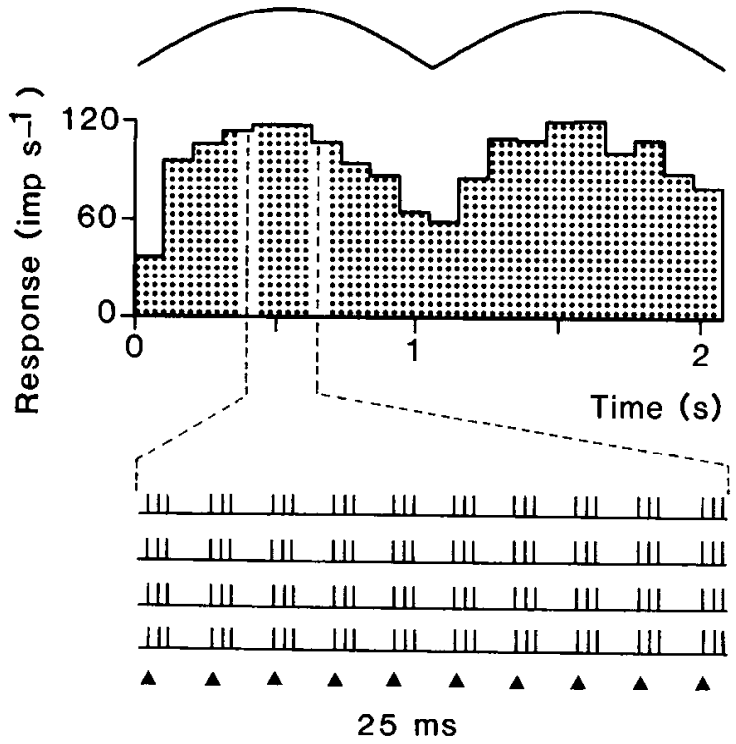

C.

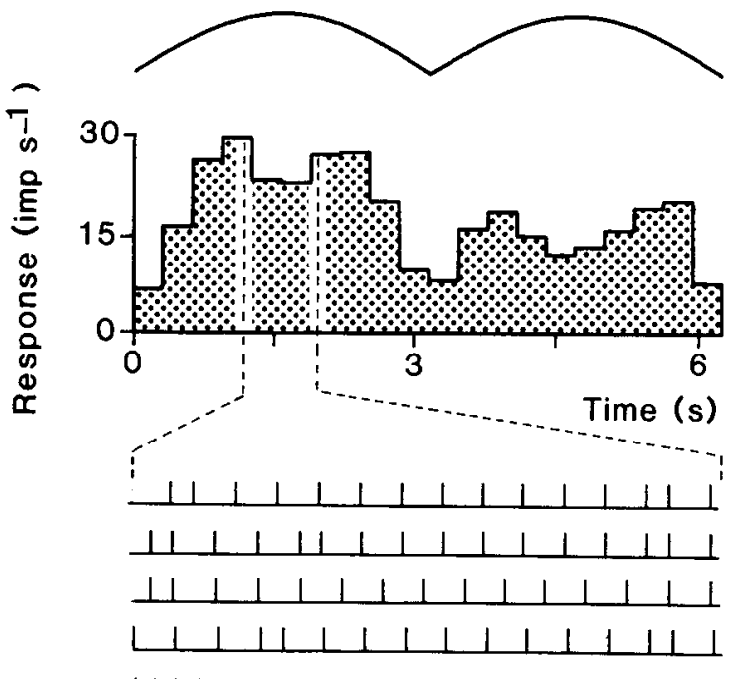

Interspike interval
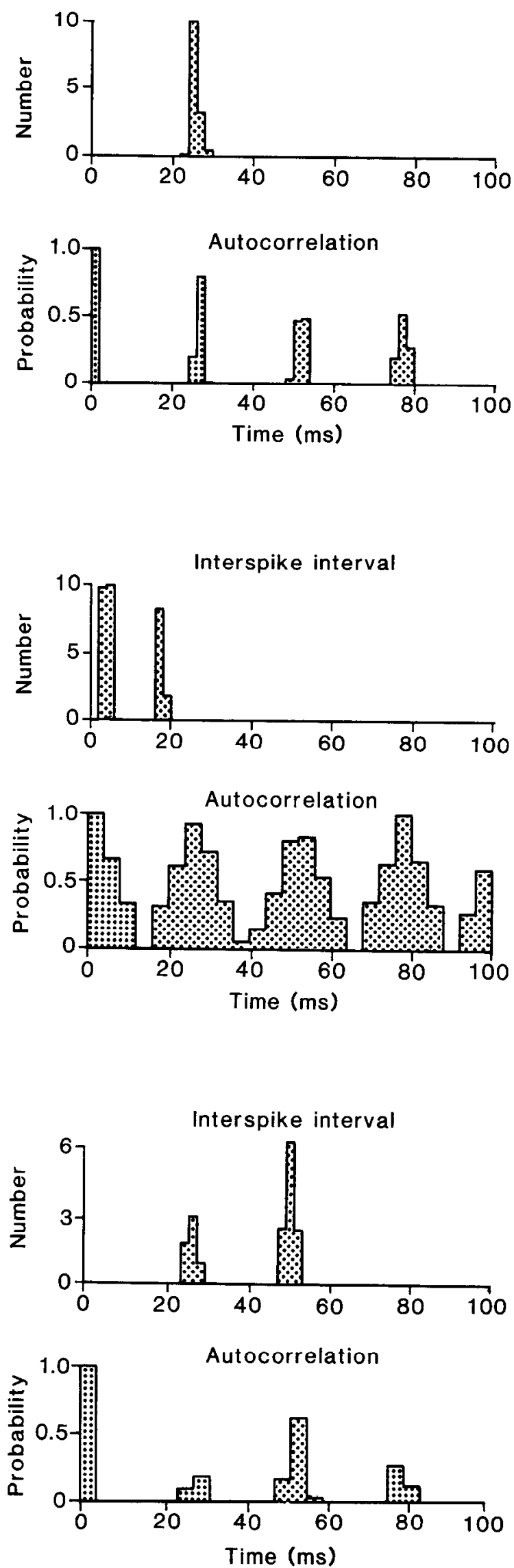

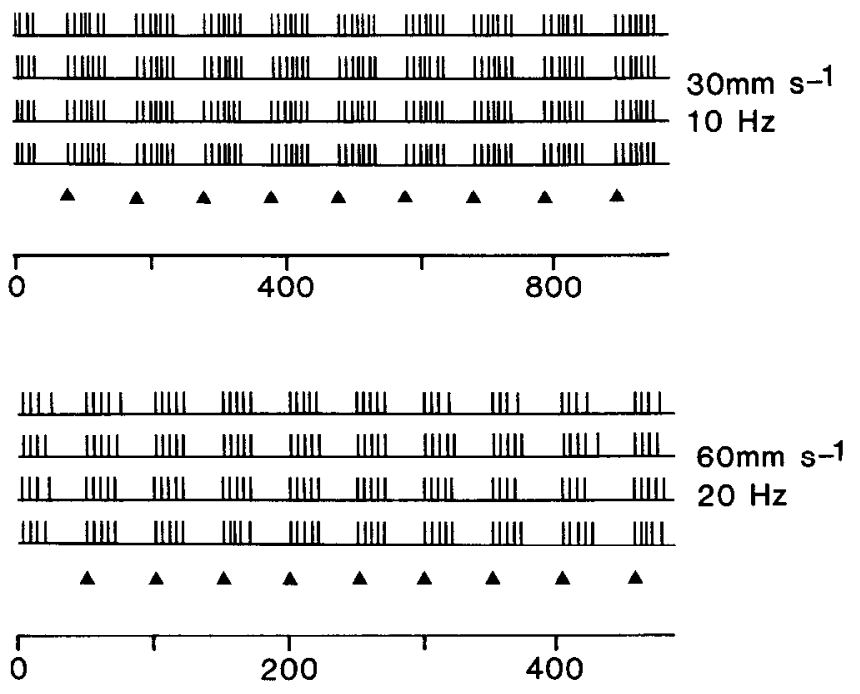

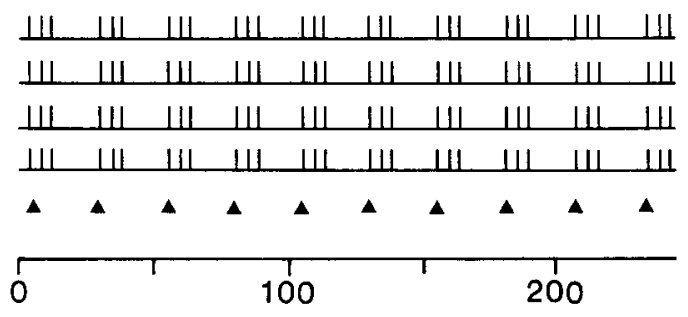

\begin{tabular}{|c|c|c|c|c|c|c|c|c|}
\hline 1 & 1 & $\perp$ & $\perp$ & 1 & $\perp$ & $\perp$ & 1 & 1 \\
\hline 1 & 1 & 1 & 1 & 1 & 1 & 1 & 1 & 1 \\
\hline 1 & $\perp$ & $\perp$ & 1 & 1 & 1 & $\perp$ & $\perp$ & $\perp$ \\
\hline 1 & 1 & 1 & 1 & & 1 & 1 & 1 & 1 \\
\hline$\Delta$ & $\Delta$ & $\Delta$ & A & $\boldsymbol{\Lambda}$ & $\Delta$ & $\Delta$ & $\Delta$ & $\Delta$ \\
\hline & & & & & & & & \\
\hline
\end{tabular}

$120 \mathrm{~mm} \mathrm{~s}^{-1}$
$40 \mathrm{~Hz}$

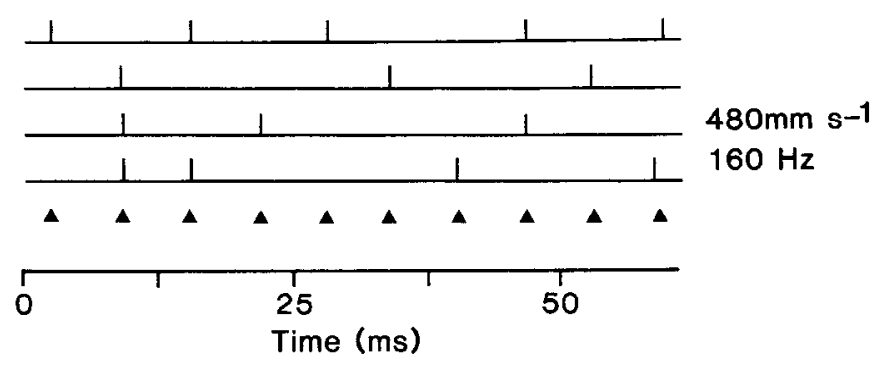

Figure 2. Phase locking in an $\mathrm{SA}$ responding to a $3 \mathrm{~mm}$ grating. The 5 groups of ensembles show the responses, at 5 different peak speeds (and corresponding peak temporal frequencies of the ridges), over the central $42^{\circ}$ of the first half cycle, where speed was constant to within $6.6 \%$ of its peak value. Note that the time base is different for each group. In each group the ensembles show the time of occurrence of action potentials for 4 consecutive cycles, and the arrowheads show the time separation between successive grating ridges $(100,50,25,12.5$, and $6.25 \mathrm{msec}$, respectively). In each case the first arrowhead has been arbitrarily aligned at the beginning of a "ridge discharge."

of Figure $1 C$ does not unambiguously specify the interval between successive ridges, but the autocorrelation function on the bottom right does. Such phase-locked responses, with an impulse occurring with only some ridges, was seen in all 3 afferent

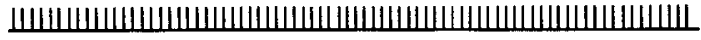

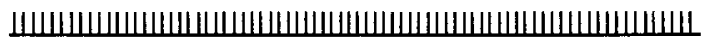

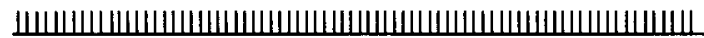

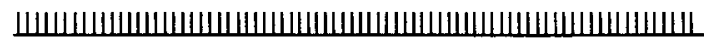
$0.75 \mathrm{~mm}$ $160 \mathrm{~Hz}$
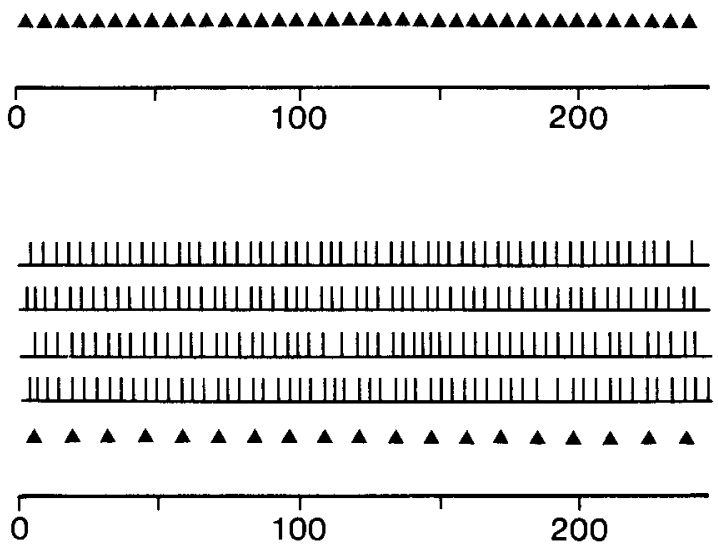

$1.5 \mathrm{~mm}$ $80 \mathrm{~Hz}$

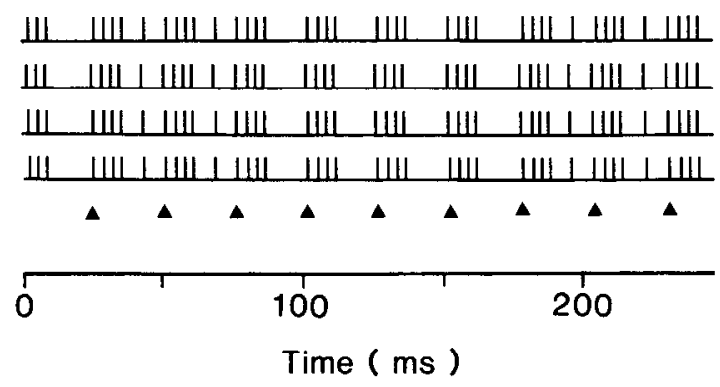

$3.0 \mathrm{~mm}$ $40 \mathrm{~Hz}$

Figure 3. Phase locking in a $\mathrm{PC}$ responding to gratings moving with a peak speed of $120 \mathrm{~mm} / \mathrm{sec}$. Three gratings were used with spatial periods of $0.75,1.5$, and $3 \mathrm{~mm}$, respectively, resulting in a peak frequency of the grating ridges of 160,80 , and $40 \mathrm{~Hz}$, respectively. Format as in Figure 2. Arrowheads show the time separation between successive grating ridges $(6.25,12.5$, and $25 \mathrm{msec}$, respectively).

populations and clearly provides the brain with information about the temporal frequency of the grating ridges.

\section{Effect of stimulus parameters on pattern of phase locking}

All afferents exhibited phase locking over the 2 central $42^{\circ}$ segments of the cycle, but the number of impulses per grating ridge varied from a fraction to as many as 12 in our experiments. How was this number affected by the stimulus parameters, that is, the spatial period of the grating, the peak speed of movement, and the peak temporal frequency of the grating ridges? Since the spatial period of the grating $(p)$, the peak temporal frequency of the ridges $(f)$, and the peak speed of movement $(s)$ are related by $p f=s$, a change in any of the stimulus parameters causes a concomitant change in a second parameter, so that it is most appropriate to examine the changes that occur when one of the stimulus parameters is held constant.

Figure 2 illustrates the responses of an SA when the spatial period of the grating remained constant at $3 \mathrm{~mm}$ and the peak speed increased from 30 to $480 \mathrm{~mm} / \mathrm{sec}$. There was a concomitant increase in the peak temporal frequency of the ridges from 10 to $160 \mathrm{~Hz}$. The separation between ridges at 10, 20, 40, 80, and $160 \mathrm{~Hz}$ was $100,50,25,12.5$, and $6.25 \mathrm{msec}$, respectively. In each case the ensembles span the same central $42^{\circ}$ of the 
cycle, so that the time base is different for each. As the spatial period of the grating remained constant at $3 \mathrm{~mm}$, the number of grating ridges moving over the receptive field during this $42^{\circ}$ portion of the cycle remained constant at approximately 9.8 (29.3/p-see Appendix). As seen in Figure 2, when the peak speed of the grating increased (peak temporal frequency of the ridges increased), the number of impulses elicited by each grating ridge decreased. This general trend was observed not only in the SAs but also in the RAs and PCs.

Figure 3 shows the responses of a $\mathrm{PC}$ when the peak speed of the grating remained constant at $120 \mathrm{~mm} / \mathrm{sec}$ and the spatial period of the grating increased from 0.75 to $3 \mathrm{~mm}$ with a concomitant decrease in the peak temporal frequency of the ridges from 160 to $40 \mathrm{~Hz}$. The separation between ridges at 160,80 , and $40 \mathrm{~Hz}$ was $6.25,12.5$, and $25 \mathrm{msec}$, respectively. Since the peak speed remained constant at $120 \mathrm{~mm} / \mathrm{sec}$, the central $42^{\circ}$ of the cycle corresponds to a time period of $0.244 \mathrm{sec}$ for all 3 cases, which therefore have the same time base. The number of grating ridges passing over the receptive field in this period $(29.3 / p)$ changed with the spatial period of the grating and was approximately $39.1,19.5$, and 9.8 for spatial periods of 0.75 , 1.5 , and $3 \mathrm{~mm}$, respectively. As is seen in the figure, the number of impulses elicited per grating ridge increased as the spatial period increased or, equivalently, as the peak temporal frequency of the ridges decreased. This general trend held not only for the PCs but also for the SAs and RAs.

The responses of an RA when the peak temporal frequency of the ridges was held constant at $40 \mathrm{~Hz}$ are illustrated in Figure 4. To maintain the peak temporal frequency constant, both the spatial period of the grating and the peak speed of movement were altered in the combinations illustrated. Since the peak speed of the grating changed, the period of time corresponding to the central $42^{\circ}$ of the cycle changed from 0.244 to 0.367 to $0.733 \mathrm{sec}$, and the time bases of the ensembles changed accordingly. Since the spatial period of the grating changed, the number of ridges passing over the receptive field during this portion of the cycle changed from approximately 9.8 to 14.7 to
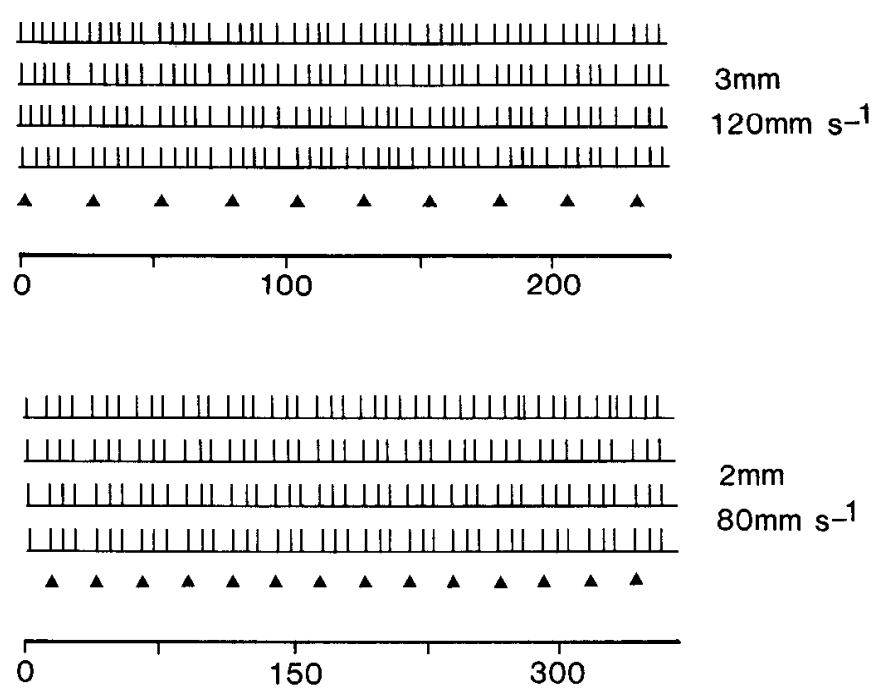

$2 \mathrm{~mm}$ $80 \mathrm{~mm} \mathrm{~s}^{-1}$

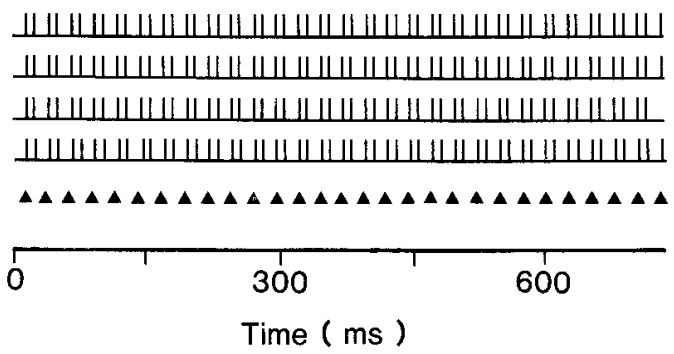

$1 \mathrm{~mm}$

$40 \mathrm{~mm} \mathrm{~s}^{-1}$

Figure 1. Phase locking in an $\mathrm{R} \Lambda$ responding to 3 combinations of grating spatial periods $(3,2$, and $1 \mathrm{~mm})$ and peak speeds of movement $(120,80$, and $40 \mathrm{~mm} / \mathrm{sec}$, respectively). In each case the peak temporal frequency of the grating ridges was $40 \mathrm{~Hz}$. Format as in Figure 2. Arrowheads show the time separation between successive grating ridges (25 $\mathrm{msec}$ in all cases).

Table 1. Percentage of SAs responding with $\boldsymbol{n}$ impulses per grating ridge

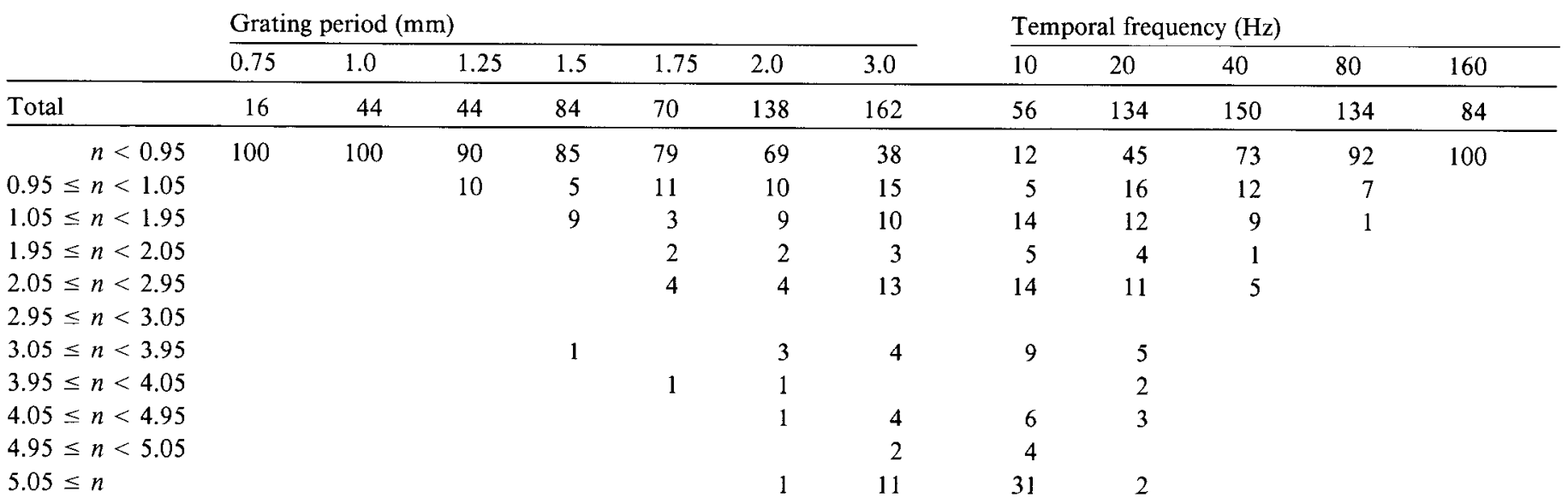

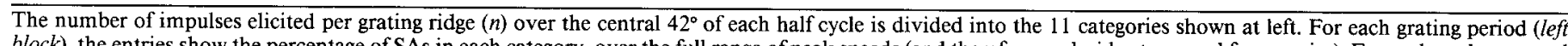

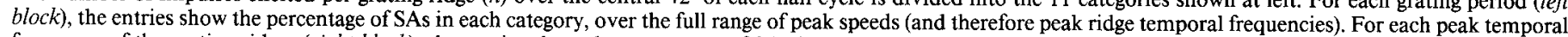

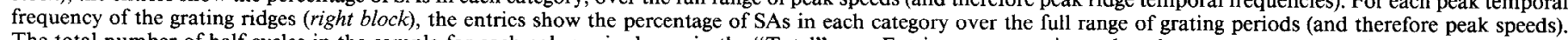

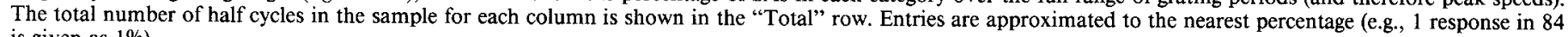
is given as $1 \%$ ). 
A.
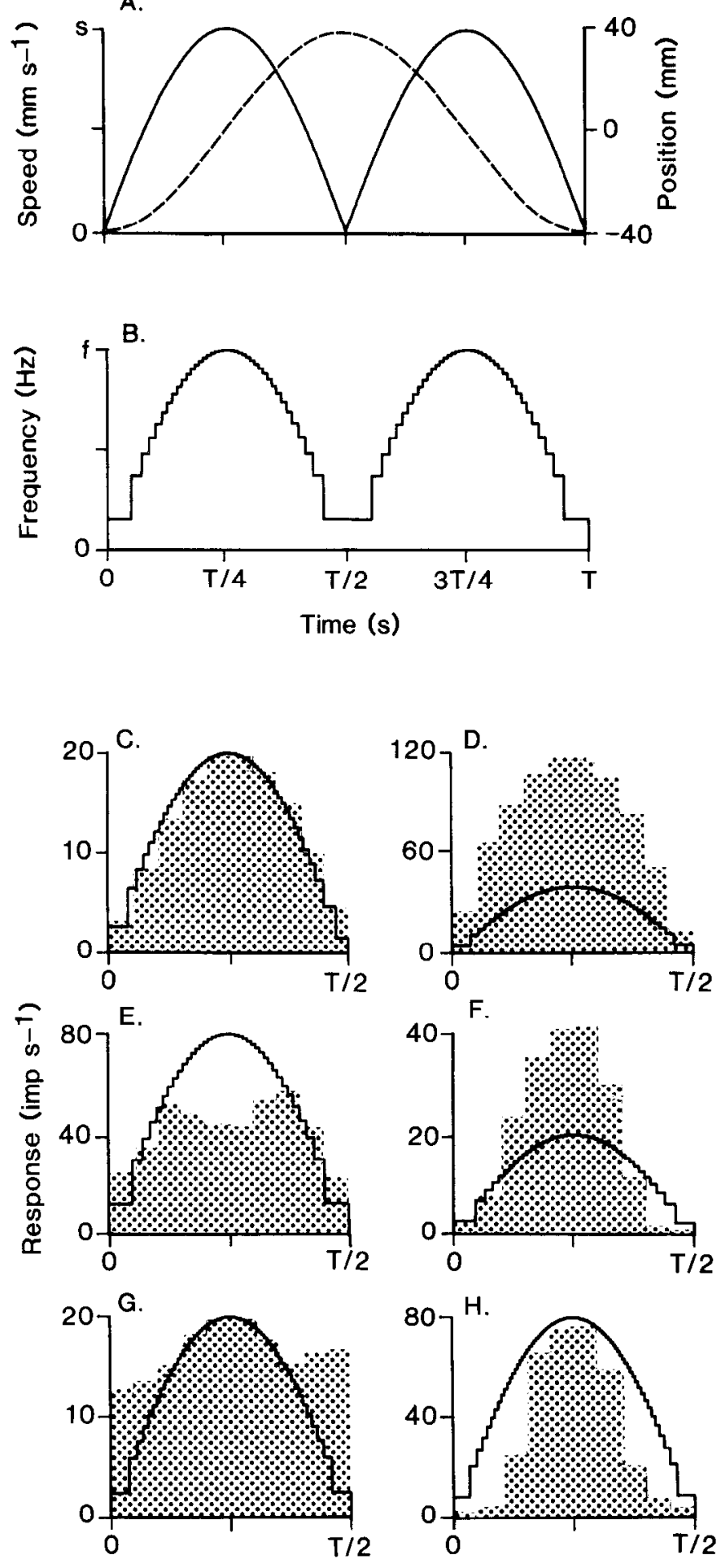

Time (s)

Figure 5. A, Position (broken) and speed (solid) profiles during a cycle of duration $T$ seconds for a grating moving with a peak speed $s(\mathrm{~mm} /$ sec). $B$, Instantaneous temporal frequency of the ridges of a $2 \mathrm{~mm}$ grating. $C-H$, Comparison of instantaneous temporal frequency of the grating ridges (solid line) with the cycle histogram (shaded area) of afferents during a half cycle. The afferent types are RA $(C)$, RA $(D)$, SA $(E), \mathrm{PC}(F), \mathrm{SA}(G)$, and $\mathrm{PC}(H)$. The grating spatial periods $(\mathrm{mm})$, pcak speeds of movement $(\mathrm{mm} / \mathrm{sec})$, peak temporal frequencies of the ridges $(\mathrm{Hz})$, and cycle lengths $(\mathrm{sec})$ were, respectively, 1.5, 30, 20, $8.378(\mathrm{C})$; $1.25,50,40,5.027(D) ; 2,160,80,1.571(E) ; 1.75,35,20,7.181(F)$; $1.25,25,20,10.053(G) ; 1,80,80,3.142(H)$. The number of cycles
29.3. The separation between ridges was $25 \mathrm{msec}$ in all 3 cases. As seen in the figure, the number of impulses elicited per grating ridge decreased with a decrease in the spatial period of grating and thus with the concomitant decrease in peak speed. This general trend held for both the SAs and the RAs. For PCs the result was more equivocal, and the number of impulses per grating ridge increased, decreased, or stayed the same depending on the 2 conditions being compared and the particular afferent.

The above results may be summarized as follows. When the spatial period of the grating was held constant, the number of impulses elicited per grating ridge, over the central $42^{\circ}$ of the cycle, decreased as the peak speed of movement increased and hence also as the peak temporal frequency of the ridges increased for all 3 afferent populations. When the peak speed of movement remained constant, the number of impulses elicited per grating ridge increased as the spatial period of the grating increased and hence as the peak temporal frequency of the ridges decreased for all 3 afferent populations. Finally, for the SA and RA populations, when the peak temporal frequency of the ridges remained constant, the number of impulses per ridge increased with an increase in the spatial period of the grating and hence with an increase in the peak speed of movement; for the PC population this result was variable.

\section{Proportion of afferents in each category of response}

Given a particular stimulus parameter (for example, a grating with a spatial period of $2 \mathrm{~mm}$ ), it is of interest to know what proportion of each of the 3 afferent populations was phaselocked with less than 1 impulse per grating ridge, between 1 and 2 impulses per grating ridge, etc. Based on the number of impulses per grating ridge $(n)$, the responses were divided into the following 11 categories: $n<0.95,0.95 \leq n<1.05,1.05 \leq n<$ 1.95 , etc., up to $4.95 \leq n<5.05,5.05 \leq n$. To calculate $n$, the mean number of impulses occurring over the central $42^{\circ}$ portion of the first half cycle was calculated, the mean being over all cycles in that run. This mean value was then divided by the number of grating ridges passing through the receptive field in that $42^{\circ}$ period $(29.3 / p)$. Similarly, a value of $n$ was calculated for the second half cycle. Since many of the responses were phase-locked with an integral number of impulses per ridge, we chose specific categories for these cases: To avoid the problem of minor computational errors, a small deviation of the response was allowed (e.g., $1: 1$ responses are defined by the category 0.95 $\leq n<1.05$ ). Table 1 shows the percentage of SA afferents falling into each of the categories. The left half of the table shows the distribution for each value of the grating spatial period, and the right half shows the distribution for cach pcak temporal frequency of the ridges. Tables 2 and 3 show the equivalent for the RA and PC populations. These categories are purely arbitrary and are shown merely to give some idea of the behavior of the 3 afferent populations. For example, it is obvious from these tables that for a grating with a spatial period of $1.5 \mathrm{~mm}$, $56 \%$ of the RA responses we studied (with the movement speeds we used) had phase-locked responses with more than 1 impulse per ridge, whereas only $10 \%$ of the SA responses we studied had more than 1 impulse per ridge.

averaged in the cycle histograms and the bin widths are, respectively, 5, $0.419 \mathrm{sec}(C) ; 15,0.251 \mathrm{sec}(D) ; 21,0.079 \mathrm{sec}(E) ; 5,0.359 \mathrm{sec}(F)$; $9,0.503 \mathrm{sec}(G) ; 22,0.157 \mathrm{sec}(H)$. 
Table 2. Percentage of RAs responding with $\boldsymbol{n}$ impulses per grating ridge

\begin{tabular}{|c|c|c|c|c|c|c|c|c|c|c|c|c|c|}
\hline & \multicolumn{7}{|c|}{ Grating period (mm) } & \multicolumn{6}{|c|}{ Temporal frequency (IIz) } \\
\hline & 0.75 & 1.0 & 1.25 & 1.5 & 1.75 & 2.0 & 3.0 & 10 & 20 & 40 & 80 & 160 & 320 \\
\hline Total & 88 & 116 & 78 & 120 & 70 & 96 & 98 & 24 & 92 & 204 & 150 & 146 & 54 \\
\hline$n<0.95$ & 73 & 59 & 55 & 38 & 18 & 15 & 2 & & 2 & 24 & 39 & 58 & 100 \\
\hline $0.95 \leq n<1.05$ & 7 & 9 & 5 & 6 & 7 & 6 & 6 & & 2 & 7 & 6 & 15 & \\
\hline $1.05 \leq n<1.95$ & 11 & 16 & 20 & 20 & 26 & 23 & 19 & & 9 & 17 & 30 & 27 & \\
\hline $1.95 \leq n<2.05$ & & 2 & 2 & 4 & 6 & & 3 & & 2 & 1 & 8 & & \\
\hline $2.05 \leq n<2.95$ & 8 & 10 & 10 & 11 & 14 & 16 & 10 & & 14 & 19 & 16 & & \\
\hline $2.95 \leq n<3.05$ & & 1 & 1 & 2 & 2 & 2 & & & 1 & 3 & & & \\
\hline $3.05 \leq n<3.95$ & 1 & 3 & 7 & 9 & 10 & 17 & 14 & & 14 & 21 & 1 & & \\
\hline $3.95 \leq n<4.05$ & & & & 2 & & 2 & 2 & & 1 & 2 & & & \\
\hline $4.05 \leq n<4.95$ & & & & 7 & 8 & 6 & 7 & 4 & 21 & 4 & & & \\
\hline $4.95 \leq n<5.05$ & & & & & 1 & & & & 1 & & & & \\
\hline $5.05 \leq n$ & & & & 1 & 8 & 13 & 37 & 96 & 33 & 2 & & & \\
\hline
\end{tabular}

Format as in Table 1.

\section{Shape of the response profile}

As stated previously, all afferents we studied responded cyclically in synchrony with the stimulus, but the shape of the response profile within a cycle showed considerable variation. The factors determining the profile shape are complex and are outlined below.

Figure $5 \mathrm{~A}$ shows the instantaneous position of the grating (broken line) and the instantaneous speed of the grating (solid line) during a single cycle of duration $T$ (in seconds). The position profile is always the same, regardless of the spatial period of the grating or of the peak speed of movement. The speed profile always has the same shape, with a scaling factor depending on the peak speed of movement $s$. To understand the response profiles of the afferents, it is convenient to commence by considering the profile of the instantaneous temporal frequency of the grating ridges passing over the receptive field. As shown in the Appendix, this is a complex function depending both on the spatial period of the grating and on the peak speed of movement. The following general description arises from the derivations in the Appendix. As an illustration, Figure $5 B$ shows the profile for a grating with a spatial period of $2 \mathrm{~mm}$. The form of Figure $5 B$ is determined by the spatial period of the grating, and the axis scaling factors are determined by the peak speed of movement. The peak speed of movement $(s)$, the peak temporal frequency of the ridges $(f)$, the spatial period of the grating $(p)$, and the temporal period of the cycle $(T)$ are governed by the relationships $s=p f$ and $s=80 \pi / T$. Thus, for example, doubling the peak speed $(s)$ doubles the peak temporal frequency of the ridges $(f)$ and halves the cycle period $(T)$. The instantaneous temporal frequency of the grating ridges is a steplike function of time: the length (abscissa) of the steps is not constant during the cycle, being largest at the points of zero speed and decreasing to a minimum at the points of maximum speed. The length of the steps (as a fraction of the cycle length) is governed by the spatial period of the grating and increases as the spatial period increases. Similarly, the height (ordinate) of the steps (as a fraction of the peak frequency) is determined by the spatial period of the grating. Around the peak speed of movement, the profile closely approximates the continuous speed profile. Near the points of zero speed, the profile differs most from the speed profile. Here, the step nature of the profile is significant, and the minimum temporal frequency of the ridges does not reach zero. This minimum temporal frequency (as a fraction of the

Table 3. Percentage of PCs responding with $\boldsymbol{n}$ impulses per grating ridge

\begin{tabular}{|c|c|c|c|c|c|c|c|c|c|c|c|c|c|}
\hline & \multicolumn{7}{|c|}{ Grating period (mm) } & \multicolumn{6}{|c|}{ Temporal frequency $(\mathrm{Hz})$} \\
\hline & 0.75 & 1.0 & 1.25 & 1.5 & 1.75 & 2.0 & 3.0 & 10 & 20 & 40 & 80 & 160 & 320 \\
\hline Total & 32 & 32 & 26 & 38 & 16 & 30 & 36 & 8 & 26 & 48 & 48 & 50 & 30 \\
\hline$n<0.95$ & 53 & 62 & 53 & 44 & 31 & 20 & 11 & & 12 & 21 & 44 & 52 & 76 \\
\hline $0.95 \leq n<1.05$ & & 3 & 4 & 8 & 6 & 13 & 20 & & & & 8 & 18 & 14 \\
\hline $1.05 \leq n<1.95$ & 32 & 19 & 11 & 24 & 19 & 33 & 14 & & 4 & 42 & 25 & 20 & 10 \\
\hline $1.95 \leq n<2.05$ & 3 & & & & 7 & & 5 & & 4 & 2 & 2 & 2 & \\
\hline $2.05 \leq n<2.95$ & 3 & 10 & 12 & 5 & 6 & 7 & 11 & 12 & 19 & 6 & 7 & 8 & \\
\hline $2.95 \leq n<3.05$ & & & 4 & & & & & & & & 2 & & \\
\hline $3.05 \leq n<3.95$ & 6 & & 8 & 11 & 7 & 13 & 8 & & 23 & 8 & 12 & & \\
\hline $3.95 \leq n<4.05$ & & & & & & & & & & & & & \\
\hline $4.05 \leq n<4.95$ & & 3 & 4 & 3 & 12 & 4 & 8 & 13 & 4 & 14 & & & \\
\hline $4.95 \leq n<5.05$ & & & & & & & & & & & & & \\
\hline $5.05 \leq n$ & 3 & 3 & 4 & 5 & 12 & 10 & 23 & 75 & 34 & 7 & & & \\
\hline
\end{tabular}

Format as in Table 1 . 
peak frequency) increases as the spatial period of the grating increases, being about $19 \%$ for a $3 \mathrm{~mm}$ grating. Naturally, all profiles discussed so far are symmetrical, being the same for movement in one direction as for movement in the reverse direction. Thus, we will only be concerned with the shape of the response profile within a half cycle (movement in 1 direction), and the question of comparison of responses for the 2 directions of movement is analyzed in detail in the following paper (Goodwin and Morley, 1987b).

If an afferent behaved like an ideal detector that responded with 1 impulse every time a grating ridge passed a specific point in the receptive field, then its response profile would mirror the profile of Figure $5 B$. Such response profiles were seldom, if ever, seen, and Figure $5 C$ illustrates a profile from the group in our data that most closely approximated such an ideal case. The solid line shows the instantaneous temporal frequency of the grating ridges, and the shaded area shows the cycle histogram of the afferent. The instantaneous frequency of the afferent was estimated by the reciprocal of the interspike interval. To average this frequency over a number of cycles, the cycle must be divided into bins. The choice of this bin width is not trivial, as too large a value will smooth and unify the response excessively, whereas with too small a value the profile will be masked by noise (experimental variation), particularly when the frequency is low and the number of cycles small. We found that the best compromise of bin width resulted when the cycle was broken up into 20 bins. As seen in Figure 5, this is particularly appropriate for those regions of the cycle where the speed tends to zero and the bin width roughly matches the step size of the interval between successive ridges.

If the afferent responded uniformly to each grating ridge, but with a multiple number of impulses per ridge, then the response profile would have the same shape as in the ideal case, with a scaling factor equal to the number of impulses per ridge. An example of a response approximating this condition is shown in Figure $5 D$, where the scaling factor is 3.

Alterations in the shape of the profile occurred either because of alterations in the peaks of the response or because of alterations in the troughs of the response or some combination of them. Figure $5 E$ illustrates an afferent for which the peak response was less than the peak temporal frequency of the ridges, thus flattening the profile; for the afferent represented in Figure $5 F$ the peak response exceeded the peak temporal frequency, thus "spiking" the profile. Similarly, Figure 5, $G$ and $H$, illustrates afferents for which the troughs (minimum response) exceeded or fell short of the temporal frequency of the ridges, thus flattening or "spiking" the profiles, respectively.

Figure 5 illustrates the basic variations that occurred in the shape of the response profiles. Some profiles of course exhibited a combination of these variations, for example, raised troughs and lowered peaks, in which case the response approached uniformity. What profile shapes were seen in the 3 afferent populations? Most of the responses in the RA population were similar to a scaled profile of the ridge temporal frequency, such as that shown in Figure $5 D$, and occasionally reflected the actual frequency, as shown in Figure $5 C$. With a peak temporal frequency of the grating ridges of $80 \mathrm{~Hz}$ or more and a grating spatial period of $0.75 \mathrm{~mm}$, the RA responses sometimes exhibited a reduced peak (Fig. $5 E$ ). Responses in the SA population were occasionally similar to a scaled profile of ridge temporal frequency, like that in Figure $5 D$, and rarely reflected the actual frequency. Most SA responses were much flatter than those of the RAs, exhibiting reduced peaks, like the profile in Figure $5 E$, or raised troughs, like the profile in Figure $5 G$, and sometimes a combination of the 2 , resulting in a ncarly flat profile. The responses of the PCs were occasionally similar to a scaled profile of ridge temporal frequency, like the profile in Figure $5 D$, and rarely reflected the actual frequency. Most commonly the PCs exhibited raised troughs, like the profile illustrated in Figure $5 G$. This was particularly exaggerated with gratings with large spatial periods, for which the profiles were similar to the flat SA responses except that, in general, the mean response was greater in the PCs than in the SAs. Extreme forms of the examples in Figure 5 were occasionally seen: For instance, the peak in Figure $5 E$ was occasionally attenuated to such a degree that the response profile contained 2 spikes (at $\mathrm{T} / 8$ and $3 \mathrm{~T} / 8$ ) in each half cycle.

The shapes of the response profiles observed do not fall into completely separate groups but, rather, make up a continuum with the illustrations in Figure 5 forming convenient points on the continuum for discussion. For this reason we have not at tempted to assess the frequency with which particular shapes occur in the different afferent populations under various stimulus conditions. A more quantitative description of profile shape requires something like a harmonic analysis of each profile, which at this point we feel is unwarranted.

\section{Discussion}

\section{Information provided by phase locking}

During the central $42^{\circ}$ of each half cycle, the speed of the grating remains constant to within $6.6 \%$ of its peak value and thus the temporal frequency of the grating ridges remains constant to within $6.6 \%$ of its peak value. Here the responses of the afferents are phase-locked to the grating ridges and hence provide precise and unambiguous information about the peak temporal frequency of the grating ridges. As can be seen from the ensembles of impulses and from the autocorrelation functions of Figure 1, this information is present regardless of the number of impulses elicited by each grating ridge.

The stimulus is completely specified if any 2 of the 3 dependent variables - spatial period, peak speed, and peak temporal frequency -are known. Phase locking provides a specification of the peak temporal frequency of the grating ridges that could be used in combination with information about another parameter obtained from the mean responses as described in the previous paper (Goodwin and Morley, 1987a). In the case of active movement, the peak speed of movement might also be assessed by activity in the proprioceptor populations or in the populations of neurons producing the movement itself. In the case of both active and passive movement, the characteristics of the movement profile could, in principle, be provided by the sequence of activation of afferents with adjacent receptive fields as suggested by Darian-Smith and Oke (1980). However, such information depends on afferents whose receptive field separation is less than the spatial period of the grating $(0.75 \mathrm{~mm}$ for our finest grating), a relatively small number according to current estimates of afferent innervation densities (Johansson and Vallbo, 1979; Darian-Smith and Kenins, 1980). Moreover, in our case the interval between successive ridges is not constant, and the brain would have to reconstruct the complex temporal profile given by equation (4) in the Appendix or else assess the sequence only over the central $42^{\circ}$ of the half cycle when the interval remains approximately constant. Thus, we feel that for a paradigm like ours, the sequence of activation of receptors is 
probably not the major contributor to determining the peak speed of movement. Another apparent cue to the peak speed of movement can be seen in Figure 2. The time duration of the $42^{\circ}$ segment over which phase locking occurs is inversely proportional to the peak speed. However, on either side of this band the speed decreases slowly and the change in phase locking is gradual, so it would be difficult for the brain to define this region with precision, and the above mechanism is unlikely to be of practical value.

In summary, phase locking provides precise information about the peak temporal frequency of the grating ridges that can be used in conjunction with other information in the afferent discharge to define the stimulus parameters. While it may be possible for the brain to characterize our gratings by simple measures such as mcan discharge rate, the abundance of information present in the temporal pattern of the impulse trains is undoubtedly also used by the brain. With complex 2-dimensional surfaces, there may well be cases when 2 different textures evoke the same mean discharge rates but can be differentiated on the basis of impulse patterns.

\section{Magnitude of the phase-locked response}

The peak temporal frequency of the grating ridges is specified by a phase-locked response regardless of the number of impulses elicited by each grating ridge. However, this magnitude of the phase-locked response varies systematically with the stimulus parameters and thus provides information about them. The number of impulses per ridge is proportional to the mean response over the $42^{\circ}$ segment of the cycle. If the response profile shape were invariant, then this would also be proportional to the mean response over a cycle. Thus, the magnitude of the phase-locked response and the mean cyclic response are closely related, at least for our stimulus. We have used the mean cyclic response, which is a simpler measure for the brain to compute, to describe the changes that occur with changes in the stimulus parameters (Goodwin and Morley, 1987a), and we feel that a detailed parametric analysis of the number of impulses per ridge is not warranted at this stage.

Two examples of the consistency of the mean cyclic response and the magnitude of the phase-locked response follow. If the number of impulses per ridge in the $42^{\circ}$ segment is $n$, then the mean response over this segment ( $R$ impulses/sec) is given by $R=n f=n s / p$. Thus, with a grating of constant spatial period $p, n$ is proportional to $R / s$. Using the approximation that the mean cyclic response is proportional to the mean response over the $42^{\circ}$ segment, we have that $n$ is proportional to (mean cyclic response) $/ s$. From Figure 7 of the previous paper (Goodwin and Morley, 1987a), when the grating spatial period remains constant, an increase in peak speed increases the mean cyclic response of the RAs but by a factor less than the increase in peak speed (slope on linear scales less than 1). Thus, on the basis of the above argument, $n$ decreases. For the SAs (Fig. 5 of Goodwin and Morley, 1987a) the mean response does not change, so that $n$ again decreases with an increase in $s$ but does so more rapidly than was the case for the RAs. These predictions were borne out by our results, and a similar analysis for the other combinations of afferents and stimulus parameters also reveals the consistency.

These results may appear to conflict with the data DarianSmith and Oke (1980) obtained using gratings moving over the fingertip at constant speed. This results, in part, from their emphasis on phase-locked responses with 1 impulse per grating ridge: However, the majority of their responses had more than or less than 1 impulse per ridge. In addition, the range of stimulus parameters used by them was smaller than that used here.

\section{The response profile}

A rigorous analysis of the factors affecting the response profile is complex. The position of the grating varies sinusoidally, the speed of movement is a rectified sinusoid, and, as shown in the Appendix, the temporal frequency of the grating ridges is a complex function of grating speed and spatial period that approximates a rectified sinusoid as the spatial period of the grating tends to zero. For a grating with a spatial period of $3 \mathrm{~mm}$ the minimum temporal frequency of the ridges is not zero but a surprisingly high $19 \%$ of the peak temporal frequency. Of the 3 groups of afferents, the RAs most commonly had profiles (over a half cycle) that were reasonably close to sinusoidal in shape. This is consistent with a receptor having a flat frequency response, reflecting the near sinusoidal temporal frequency of the grating ridges. As far as can be determined from the literature, responses of RAs to probes vibrating sinusoidally at constant amplitude also display essentially flat frequency response curves-either high-pass filters or band-pass filters with the cutoff frequencies beyond our range of interest (Freeman and Johnson, 1982; Johansson et al., 1982). The SAs often showed a peak response less than that expected on the basis of the response during the rest of the cycle. This is consistent with a receptor having a low-pass filter characteristic, failing to respond to the higher temporal frequencies of the ridges around the peak. Available evidence suggests that SAs have low-pass filter characteristics for vibratory sinusoids, but the data are scanty, particularly at the high frequencies (Freeman and Johnson, 1982; Johansson et al., 1982). With many SAs the responses as the speed approached zero were much greater than would be expected on the basis of ridge temporal frequency, and the profiles were much flatter than might have been anticipated. This can be attributed to the "static" response of SAs. However, a grating moving sinusoidally over the receptive field is not equivalent to a sinusoidally vibrating probe and such data cannol explain all aspects of the profiles. The vigorous response of many PCs in the region of low speed is striking. Vibratory data (Freeman and Johnson, 1982; Johansson et al., 1982; Bolanowski and Zwislocki, 1984) show a high-pass filter characteristic for PCs, which therefore might not be expected to respond at the speeds approaching zero, where the temporal frequency of the ridges also approaches zero. On the contrary, in this region many PCs responded vigorously with many impulses accompanying each ridge passage. Apparently the PCs were responding to each ridge edge (an edge contains a whole spectrum of frequencies). In summary, simple comparison of our stimulus and a vibratory probe is not valid and could be misleading. A rigorous comparison would require a complete spatiotemporal model of the skin mechanics and the receptor mechanisms and is beyond the scope of this paper.

For similar reasons we have not attempted to model the variation of the response profiles with variations in the stimulus parameters. Rather, we have studied the effects on specific aspects of the response such as mean cyclic response, phase locking, etc., which, of course, reflect general changes in the profile and which are probably of more direct use to the brain. Nor have we analyzed the nature of phase locking outside the region of approximately constant speed. During such portions of the sinusoid, the speed is changing continuously and an additional 
variable, namely, acceleration, is introduced. From the range of profiles seen (e.g., Fig. 5) it is clear that the nature of any phase locking in these regions is complex. Here each region of relatively constant speed and acceleration is small and the amount of data provided is insufficient for statistically significant analysis. We feel that the effects of acceleration should be studied using a different pattern of movement, for example, one with constant acceleration.

\section{Appendix_Instantaneous temporal frequency of grating ridges}

Consider a grating, with spatial period $p$ (in $\mathrm{mm}$ ), moving sinusoidally over a receptive field with a peak-to-peak amplitude of $80 \mathrm{~mm}$ and a cycle period of $T$ seconds. Let the position, velocity, and speed at any instant of time be $x(\mathrm{~mm}), \dot{x}(\mathrm{~mm} / \mathrm{sec})$, and $|\dot{x}|(\mathrm{mm} / \mathrm{sec})$, respectively, and let the peak speed be $s(\mathrm{~mm} / \mathrm{sec})$. The position may be described by

$$
x=-40 \cos (2 \pi t / T)
$$

The phase angle in equation (1) is chosen to satisfy our convention of a cycle commencing at one extreme position of the grating. It follows that

$$
\begin{aligned}
\dot{x} & =(80 \pi / T) \sin (2 \pi t / T) \\
& =s \sin (2 \pi t / T) \\
|\dot{x}| & =s|\sin (2 \pi t / T)|, \quad \text { where } s=80 \pi / T
\end{aligned}
$$

In Figure $5 A$ the broken line shows the instantaneous position $x$ during a cycle and the solid line shows the speed $|\dot{x}|$.

Now suppose that at time $t$ seconds a grating ridge passes over a spccific point in the receptive ficld, and at time $t+i \sec$ the next ridge passes over that same point. Since successive ridges are separated by $p$ $(\mathrm{mm})$, the relationship between the time interval $i$ elapsing between successive ridges, the spatial period $p$ of the grating, and the peak speed of movement $s$ is given by equation (1) as

$$
\begin{aligned}
p & =40|\cos (s t / 40)-\cos [s(t+i) / 40]| \\
& =80|\sin [(2 s t+s i) / 80] \sin (s i / 80)|
\end{aligned}
$$

In the following analysis the variables of interest, as a function of time, are the time interval between successive ridges, $i$ (sec), and its reciprocal $(1 / i)$, which we call the instantaneous temporal frequency of the ridges $r$ (in $\mathrm{Hz}$ ). Figure $5 B$ shows the instantaneous temporal frequency of the ridges $(r)$, during a cycle, for a grating with a spatial period of $2 \mathrm{~mm}$ derived from cquation (4). Since $r$ is only defined between successive ridges, the function is steplike. The length (abscissa) of the steps and the height (ordinate) of the steps increase towards the points of zero speed ( $t=0$ and $t=T / 2)$ and decrease towards the points of maximum speed $(t=T / 4$ and $t=3 T / 4)$. The form of the curve is the same for all spatial periods of the grating $(p)$ and all peak speeds $(s)$. Changing $s$ changes only the scaling factors of the 2 axes, i.e., the value of $T$ and the value of $f$. Changing $p$ changes the scaling factor of the ordinates, i.e., the value of $f$, and also changes the size of the steps (as a fraction of $T$ and $f$ ). As can be seen from equation (4) the relationship between $r, p$, and $s$ is cumbersome. However, several simple approximations are apparent.

\section{Region of constant speed}

During the 2 portions of the cycle when the speed is maximum $(t=T / 4$ and $t=3 T / 4)$, the speed remains approximately constant and is within $6.6 \%$ of $s$ for $42^{\circ}$ of the cycle. Thus, in both of these regions, the temporal frequency of the grating ridges remains constant for $42^{\circ}$ of the cycle to within $6.6 \%$ of its peak value $f$. Obviously, the constants $s$ and $f$ are related by

$$
s=p f
$$

Equation (6) can also be derived from equation (5) using the approximation $\sin (s i / 40) \simeq s i / 40$, which demonstrates that the approximation of equation (6) is valid only when $i$ is a small fraction of the cycle period. (In our experiments, $s i / 40$ had a maximum value of $4.3^{\circ}$ in this region.)

\section{Spatial period $\mathrm{p}$ tends to zero}

As $p \rightarrow 0, i \rightarrow 0$. Substituting $p \simeq|d x|$ and $i \simeq d t$ in equation (2) gives

$$
\begin{aligned}
d x & =s \sin (2 \pi t / T) d t \\
\therefore p & =\operatorname{si}|\sin (2 \pi t / T)| \\
\therefore r & =1 / i=\sin |(2 \pi t / 1)| s / p
\end{aligned}
$$

from equation (6)

$$
=f|\sin (2 \pi t / T)|
$$

In other words, the instantaneous temporal frequency of the grating ridges is a rectified sinusoid in phase with the speed of the grating movement and with a peak value $f=s / p$ but only if the period of the grating is vanishingly small.

\section{Minimum ridge frequency}

For nonzero spatial periods of the grating, the instantaneous temporal frequency of the ridges differs most from the sinusoidal profile at the points where the speed of movement is zero, i.e., $t-0$ and $t-T / 2$. For small si/80 (in our experiments $s i / 80 \leq 11.2^{\circ}$ at these points), at $t=0$ and $t=T / 2$, equation (5) can be approximated by

$$
\begin{aligned}
p & \simeq 80(s i)^{2} /(80)^{2} \\
\therefore r_{\min } & =1 / i \\
& =s / \sqrt{80 p}
\end{aligned}
$$

Thus, this minimum temporal frequency of the ridges, which is zcro for gratings with vanishingly small spatial periods, depends both on the spatial period of the grating and on the peak speed of movement. Expressing this minimum ridge frequency as a fraction of the maximum temporal frequency of the ridges occurring in the cycle gives, from equations (8) and (6),

$$
r_{\min } / r_{\max }=\sqrt{p / 80}
$$

It is interesting that this ratio depends only on the grating spatial period and not on the peak speed of movement: It is greatest in our experiments for gratings with a spatial period of $3 \mathrm{~mm}$, where the minimum temporal frequency of the grating ridges is a surprisingly high $19 \%$ of the peak frequency.

\section{Number of ridges in constant speed region}

For a $42^{\circ}$ segment of the cycle centered on $t=T / 4$ or $t=3 T / 4$, the number of grating ridges passing through the receptive field can be calculated as

$$
\begin{aligned}
\text { duration of } 42^{\circ} \text { segment } & =42 T / 360 \mathrm{sec} \\
\therefore \text { number of ridges in segment } & =42 \mathrm{fT} / 360 \\
\text { from equations (6) and (3) } & =28 \pi / 3 p
\end{aligned}
$$

\section{Turnaround artifact}

When the direction of movement of the grating reverses $(t=0$ and $t=$ $T / 2$ ), the time between successive ridges passing a point in the receptive field may differ from that calculated at these points without allowing for a reversal-equation (8). If the direction reverses just before a ridge passes the point, then the previous ridge will again pass the point in the reverse direction, and the interval between successive occurrences of this ridge at the point will be nearly double that estimated in equation (8). On the other hand, if direction reversal occurs just after a ridge has passed the point, then the same ridge will again pass the point in the reverse direction, and the time intcrval between these 2 passages will be less than that calculated in equation (8). In the analysis this artifact cannot be taken into account, as we have no way of defining the absolute position of the ridges with respect to such a point in the receptive field, which in any event is an abstraction. However, it should be borne in mind that this reversal effect could result in unexpectedly large or small intervals between impulses at the turnaround points.

\section{References}

Bolanowski, S. J., Jr., and J. J. Zwislocki (1984) Intensity and frequency characteristics of pacinian corpuscles. I. Action potentials. J. Neurophysiol. 51: 793-811.

Darian-Smith, I., and P. Kenins (1980) Innervation density of mech- 
anoreceptive fibres supplying glabrous skin of the monkey's index finger. J. Physiol. (Lond.) 309: 147-155.

Darian-Smith, I., and L. E. Oke (1980) Peripheral neural representation of the spatial frequency of a grating moving across the monkey's finger pad. J. Physiol. (Lond.) 309: 117-133.

Ferrington, D. G., and M. Rowe (1980) Differential contributions to coding of cutaneous vibratory information by cortical somatosensory areas I and II. J. Neurophysiol. 43: 310-331.

Freeman, A. W., and K. O. Johnson (1982) Cutaneous mechanoreceptors in macaque monkey: Temporal discharge patterns evoked by vibration, and a receptor model. J. Physiol. (Lond.) 323: 21-41.

Gardner, E. P., and W. A. Spencer (1972) Sensory funneling. I. Psychophysical observations of human subjects and responses of cutaneous mechanoreceptive afferents in the cat to patterned skin stimuli. J. Neurophysiol. 35: 925 -953.

Goodwin, A. W., and J. W. Morley (1987a) Sinusoidal movement of a grating across the monkey's fingerpad: Representation of grating and movement features in afferent fiber responses. J. Neurosci., 7: $2168-2180$.

Goodwin, A. W., and J. W. Morley (1987h) Sinusoidal movement of a grating across the monkey's fingerpad: Effect of contact angle and force of the grating on afferent fiber responses. J. Neurosci., 7: 21922202.

Johansson, R. S., and A. B. Vallbo (1979) Tactile sensibility in the human hand: Relative and absolute densities of four types of mechanoreceptive units in glabrous skin. J. Physiol. (Lond.) 286: 283300.

Johansson, R. S., U. Landström, and R. Lundström (1982) Responses of mechanoreceptive afferent units in the glabrous skin of the human hand to sinusoidal skin displacements. Brain Res. 244: 17-25.

Johnson, K. O., I. Darian-Smith, C. LaMotte, B. Johnson, and S. Oldfield (1979) Coding of incremental changes in skin temperature by a population of warm fibers in the monkey: Correlation with intensity discrimination in man. J. Neurophysiol. 42: 1332-1353.

LaMotte, R. H., and V. B. Mountcastle (1975) Capacities of humans and monkeys to discriminate between vibratory stimuli of different frequency and amplitude: A correlation between neural events and psychophysical measurements. J. Neurophysiol. 38: 539-559.

Orban, G. A., H. Kato, and P. O. Bishop (1979) End-zone region in receptive fields of hypercomplex and other striate neurons in the cat. J. Neurophysiol. 42: 818-832.

Perkel, D. H., and T. H. Bullock (1968) Neuronal coding. Neurosci. Kes. Program Bull. 6: 221-348.

Phillips, J. R., and K. O. Johnson (1981) Tactile spatial resolution. II. Neural representation of bars, edges, and gratings in monkey primary afferents. J. Neurophysiol. 46: 1192-1203.

Poggio, G. F., and L. J. Viernstein (1964) Time series analysis of impulse sequences of thalamic somatic sensory neurons. J. Neurophysiol. 27: 517-545.

Rose, J. E., J. F. Brugge, D. J. Anderson, and J. E. Hind (1967) Phaselocked response to low frequency tones in single auditory nerve fibers of the squirrel monkey. J. Neurophysiol. 30: 769-793.

Talbot, W. H., I. Darian-Smith, H. H. Kornhuber, and V. B. Mountcastle (1968) The sense of Aluter-vibration: Comparison of the human capacity with response patterns of mechanoreceptive afferents from the monkey hand. J. Neurophysiol. 31: 301-334. 